\title{
Réforme éducative au Laos : vers la prise en compte du genre et de l'ethnicité
}

Educational reform in Laos: Towards the taking into account of gender and ethnicity

Reforma educativa en el Laos: hacia la consideración del género y de la etnicidad

\section{Kongsy Chounlamany}

Traducteur : Sylvaine Herold

\section{OpenEdition}

\section{Journals}

Édition électronique

URL : https://journals.openedition.org/ries/6589

DOI : $10.4000 /$ ries. 6589

ISSN : 2261-4265

Éditeur

France Education international

\section{Édition imprimée}

Date de publication : 1 septembre 2018

Pagination : 115-124

ISBN : 978-2-85420-619-7

ISSN : 1254-4590

\section{Référence électronique}

Kongsy Chounlamany, "Réforme éducative au Laos : vers la prise en compte du genre et de l'ethnicité », Revue internationale d'éducation de Sèvres [En ligne], 78 | septembre 2018, mis en ligne le 01 septembre 2020, consulté le 25 juin 2021. URL : http://journals.openedition.org/ries/6589 ; DOI : https://doi.org/10.4000/ries.6589 


\section{Réforme éducative au Laos : vers la prise en compte du genre et de l'ethnicité*}

\section{Kongsy Chounlamany \\ Université nationale du Laos}

La République démocratique populaire lao entre dans une nouvelle phase de développement socio-économique. L'objectif ambitieux du gouvernement est de mettre en place d'ici 2020 une structure industrielle moderne adaptée. Atteindre cet objectif implique nécessairement la mise à niveau des ressources humaines au niveau national, comme cela est prévu dans les directives sur le rôle et la qualité de l'éducation pour la nouvelle période.

Le ministère de l'éducation et des sports du Laos ainsi que la plupart des pédagogues au niveau national s'efforcent d'identifier les conditions et facteurs les plus propices pour améliorer la qualité du développement éducatif au Laos.

Cet article s'intéresse aux conséquences des relations de pouvoir, à l'intersection des enjeux ethniques, éducatifs et de genre, provenant des croyances, de la culture et des traditions séculaires du peuple lao - tel que le modèle de famille patriarcal -, qui se manifestent dans les structures sociales. Au quotidien, ces structures génèrent des comportements genrés et ethniques répétés chez les hommes comme chez les femmes, créant des rapports de force inégaux entre les sexes et entre les différents groupes ethniques. De plus, dans les régions reculées, il existe encore des limites et des contraintes multiples au développement des opportunités éducatives, en raison de l'éloignement géographique, du manque d'infrastructures de transport et du faible niveau de revenu des familles.

La disparité des opportunités éducatives selon le genre et l'ethnicité, qui se manifeste dans le fait que parmi le nombre important d'élèves qui décrochent scolairement figure une majorité de filles et d'enfants issus des minorités ethniques, a pour effet que de moins en moins de femmes qualifiées participent au développement socio-économique du pays ou se trouvent à des fonctions de pouvoir dans le secteur public. Le fossé existant entre les sexes et l'importance du pouvoir masculin sont également clairement visibles dans les différences de statut social au sein de l'organisation étatique.

Cet article propose un état des lieux des inégalités ethniques et de celles liées au genre aux niveaux local et national au Laos. Nous avons pour cela recueilli des données issues de différents documents officiels, relatives à

\footnotetext{
* Article traduit par Sylvaine Herold.
} 
l'éducation, aux enfants, aux établissements scolaires, au sexe et à l'appartenance ethnique, en cherchant à répondre aux questions suivantes : quel est l'état de l'accès à l'éducation des enfants au Laos ? Quelle attention est accordée aux minorités sexuelles et ethniques dans ce domaine?

\section{ÉLÉMENTS DE CONTEXTE}

Pour notre analyse, nous avons eu recours à des documents synthétiques rassemblant des données de différents types.

Au Laos, la moitié de la population a moins de 23,5 ans (Bureau de la statistique du Laos, 2016), ce qui donne au pays une opportunité démographique d'accélérer son développement économique. Le taux de croissance de la population est de 1,4 et son taux de fertilité de 2,7 (Unescap, 2016, in Unesco, UNDP, IOM, UN-Habitat, 2017). $59 \%$ de la population du pays vit toujours dans les zones rurales (ibid.), où le taux de pauvreté est 2,9 fois plus élevé que celui des zones urbaines (République démocratique populaire lao, 2015). L'agriculture est largement pratiquée comme activité de subsistance et les conditions de production des agriculteurs sont généralement médiocres (Ifad 2014, 2011, in Unesco, UNDP, IOM, UN-Habitat, 2017). Par conséquent, cette pauvreté rurale explique l'importance des migrations internes et externes

La République démocratique populaire lao est l'un des pays pauvres d'Asie du Sud-Est et c'est probablement le pays le plus diversifié de la région, au plan ethnique. Près de 6,5 millions de personnes vivent au Laos, dont 3,2 millions de femmes. Les conditions de vie parmi les groupes ethniques qui composent le pays ne sont pas toujours propices à une bonne nutrition. La population rurale représente $67 \%$ de la population nationale, dont $59 \%$ vit dans des zones rurales avec accès routier. On distingue quatre grandes familles ethnolinguistiques : les Lao-Tai (67 \% de la population), les Môn-Khmer (21\%), les Hmong-Lu Mien ( $8 \%$ ) et les Sino-Tibétains (3\%). Ces catégories comprennent en outre 49 ethnies distinctes et quelque 200 sous-groupes ethniques lao (King et van de Walle, 2006).

Dans son préambule, la Constitution de 1991 de la République démocratique populaire lao insiste sur la nécessité d'éduquer la population. Son article 19 stipule ainsi que "l'État affirme l'importance du développement de l'éducation en même temps que de l'édification d'une nouvelle génération de bons citoyens $»$.

Le Plan d'action national en faveur de l'éducation pour tous (2003-2015) a fixé trois objectifs majeurs : l'accès équitable à l'école, l'amélioration de la qualité et de la pertinence et le renforcement de la gestion de l'éducation pour l'éducation formelle et non formelle à tous les niveaux. Ce plan a cherché à atteindre ces objectifs en : mettant en œuvre l'éducation primaire universelle; ciblant les groupes de population défavorisés dans les zones rurales et urbaines; promouvant la participation communautaire à l'éducation de base et à 
l'alphabétisation au niveau local; améliorant la pertinence et la qualité de l'éducation de base grâce à des opportunités d'apprentissage pour les enfants, les jeunes et les adultes. Ce plan d'action national concernait l'éducation et la protection de la petite enfance, l'enseignement primaire, l'enseignement secondaire inférieur ainsi que l'éducation non formelle et la formation professionnelle.

Administrativement, le pays est divisé en 17 provinces (outre la capitale), 148 districts et 8507 villages (Recensement de la population du Laos, 2015). Ces dix dernières années, le pays a de plus été subdivisé en 70 districts non pauvres, 25 districts pauvres et 47 districts extrêmement pauvres, sur la base de différents critères de revenu, éducatifs et d'infrastructures physiques. Compte tenu du lien avéré entre éducation et pauvreté, la Stratégie nationale de croissance et d'éradication de la pauvreté [National Growth and Poverty Eradication Strategy: NGPES] semble être une politique pertinente pour un développement éducatif axé sur la lutte contre la pauvreté. Les 47 districts les plus pauvres reçoivent ainsi le plus d'attention, notamment de la part des donateurs internationaux qui ont contribué à l'élaboration de cette politique (King et Van de Walle, 2010).

Le Laos est un pays où l'on trouve à la fois une organisation sociale matrilinéaire et patrilinéaire. C'est l'un des rares pays au monde où la résidence matrilocale, l'héritage matrilinéaire et la descendance et la parenté bilinéaires existent encore pour un grand nombre de femmes, notamment les femmes de la tradition Lao Loum.

Les minorités ethniques (c'est-à-dire non lao-tai) sont particulièrement défavorisées au sein de la population rurale, en raison de facteurs comme l'exclusion sociale, les déplacements de villages, les disparités entre les sexes et le manque d'accès à l'éducation (Ifad 2014, 2011, in Unesco, UNDP, IOM, UN-Habitat, 2017). Les non Lao-Tai sont deux fois plus susceptibles que les Lao-Tai de vivre dans la pauvreté, et un tiers des filles non lao-tai en zones rurales n’ont jamais fréquenté l'école, contre moins d'un dixième des filles lao-tai. Des disparités existent également entre les différents groupes minoritaires : en zones rurales, $56 \%$ des garçons môn-khmers et $53 \%$ des filles âgées de 6 à 10 ans sont inscrits à l'école primaire, contre seulement $36 \%$ des garçons sino-tibétains et $30 \%$ des filles (King et Van de Walle, 2010).

Les migrants internes constituent une population importante au Laos. Environ $17 \%$ de la population étaient considérés en 2015 comme des « migrants définitifs ", bien que ce chiffre comprenne des personnes migrantes étrangères (Bureau de la statistique du Laos, 2016). Le Département des affaires économiques et sociales des Nations Unies (UNDESA, 2017) estime qu'environ 45500 ressortissants étrangers résidaient en 2017 en République démocratique populaire lao en 2017, ce qui ne représenterait qu'une très faible part des $17 \%$ de migrants. En comparaison, un peu plus de 1,3 million de citoyens lao résidaient en 2017 à l'étranger (UNDESA, 2017), ce qui représente environ $18 \%$ de la population.

La principale destination des migrations rurales, en particulier en provenance de la province de Vientiane et du nord du pays, est la capitale, Vientiane, 
qui compte la plus grande part de migrants à vie parmi toutes les villes du pays : quatre personnes sur dix vivant à Vientiane étaient en 2016 des migrants et la densité de population y est huit fois supérieure à la moyenne nationale (Bureau de la statistique du Laos, 2016).

Le gouvernement laotien met actuellement l'accent sur le développement des ressources humaines, en tant que facteur clé de développement socioéconomique du pays. Mais, à cet égard, le pays doit faire face à de nombreux défis ayant trait à la pauvreté, à la langue, à la culture et à la situation géographique. L'enseignement a lieu le plus souvent dans les villages et les villes du centre, alors qu'environ $80 \%$ de la population vit dans des villages ruraux éloignés (Fox, 2003), où beaucoup ne parlent pas la langue d'enseignement, le lao. Dès lors, rassembler l'ensemble des enfants pour les scolariser a été, et demeure, une tâche complexe, en particulier s'agissant des enfants des régions éloignées, dont la langue maternelle n'est pas le lao.

\section{DÉVELOPPEMENT ÉDUCATIF AU LAOS}

Le gouvernement du Laos s'efforce d'atteindre un double objectif de sortie d'ici 2020 du statut qui le classe parmi les pays les moins avancés et de réalisation d'ici 2015 les objectifs du Millénaire pour le développement. Afin de respecter les engagements de l'Éducation pour tous, le pays a développé une Stratégie nationale de réforme du système éducatif (2006-2015) qui appelait à un changement systématique afin de permettre au ministère de l'éducation et des sports d'améliorer son efficacité quant à la réalisation de ses engagements. Dans le cadre de cette stratégie, le gouvernement a initié la réorganisation de la structure du ministère de l'éducation et des sports afin d'améliorer le processus de mise en ouvre du développement éducatif (Ministère de l'éducation et des sports, 2015).

L'aspect le plus préoccupant du ralentissement actuel du développement de l'enseignement primaire est que les enfants ne fréquentant pas encore l'école primaire proviennent de manière disproportionnée des ménages à faible revenu, des groupes ethniques minoritaires et des zones rurales éloignées. Les taux nets d'inscription au primaire vont de plus de $80 \%$ pour les enfants non pauvres (garçons et filles) dans les zones urbaines à 45-55\% pour les groupes ethniques minoritaires dans les zones rurales - les filles issues de minorités ethniques et de familles rurales pauvres étant particulièrement défavorisées.

\section{GENRE ET ETHNiCité}

Le ministère de l'éducation et des sports s'est fixé des objectifs en matière de promotion des femmes dans sa stratégie nationale, notamment le fait d'accroître l'accès des filles à l'école pour atteindre en 2015 l'égalité avec les 
garçons : il s'agissait d'atteindre un taux de scolarisation brut de $98 \%$ dans l'enseignement primaire, de $75 \%$ dans l'enseignement secondaire et de $50 \%$ dans l'enseignement supérieur et à l'université. Il était en outre prévu qu'au moins un tiers de femmes occupe des postes de décision au sein du ministère (Lao Women Union, 2015).

Mais les inégalités en matière d'éducation se manifestent de manière criante, si l'on s'intéresse à la proportion de jeunes n'ayant jamais fréquenté l'école. Bien que l'entrée à l'école soit généralement tardive au Laos, dans l'ensemble, $10 \%$ des enfants âgés de 10 à 16 ans ne sont jamais allés à l'école, et il existe des différences notables selon le sexe, l'appartenance ethnique et le lieu de résidence. Dans les zones rurales, 34,3\% des filles non lao-tai et 6,0\% des filles lao-tai ne sont jamais allées à l'école. Les chiffres correspondants pour les garçons en zones rurales sont de $17,2 \%$ et 3,8 \% - des différences vraiment immenses même au sein des zones rurales (King et van de Walle, 2010).

La pauvreté accentue encore les écarts, même parmi les filles : en zones rurales, 39,8 \% des filles non lao-tai et 10,6 \% des filles lao-tai issues de familles pauvres n'ont jamais fréquenté l'école, contre $28,4 \%$ des filles non lao-tai et $4,2 \%$ des filles lao-tai issues de familles non pauvres.

Il apparaît ainsi clairement que le fait de simplement parvenir à scolariser les enfants demeure encore un défi crucial au Laos - tout comme il semble évident que les efforts devraient être ciblés sur les enfants issus des minorités ethniques et des ménages ruraux pauvres.

Dans leur étude, Jeffery H. Marshall et Emiko Naka (2016) soulignent que les groupes ethniques, en particulier môn-khmer et sino-tibétain, sont beaucoup plus susceptibles de citer la raison "pas intéressés " comme motif de ne pas inscrire leurs enfants à l'école : environ $70 \%$ des ménages issus de ces groupes choisissent cette réponse. Deux enquêtes relativement récentes (Enquête sur les dépenses et la consommation au Laos 2012-13 [Lao Expenditure and Consumption Survey: LECS] et Enquête sur la population active 2010 [Labour Force Survey: LFS] fournissent un aperçu des raisons données de ne pas inscrire les enfants de 10-14 ans à l'école. La réponse " pas intéressés » est la plus fréquemment citée. Cependant, la prévalence de la réponse "pas intéressés » parmi les groupes ethniques ne semble pas s'expliquer par un « décalage » culturel ou par des préoccupations concernant la langue de scolarisation ou l'adéquation du personnel enseignant. Presqu'aucun ménage n'a cité la «langue » comme raison de non inscription de ses enfants à l'école et le seul groupe à citer des inquiétudes au sujet des enseignants a été le groupe Hmong-Lu Mien, parmi lequel environ $30 \%$ des ménages n'ayant jamais inscrit leurs enfants à l'école ont cité des préoccupations concernant l' "absence d'enseignants ».

Ces auteurs ont également montré que l'ethnicité est une variable très importante de l'éducation au Laos, au vu des résultats significatifs de la fréquence d'enfants parlant lao à la maison (selon l'évaluation de lecture précoce [Early Grade Reading Assessment: EGRA]) ou des très grandes différences entre les 
Lao-Tai et les groupes ethniques lors des tests d'évaluation des résultats d'apprentissage des élèves [National Assessment of Student Learning Outcomes : ASLO]. La variable mesurant l'appariement ethnique (c'est-à-dire le fait que l'élève et l'enseignant soient issus du même groupe ethnique) n'est significative dans aucune des estimations, bien qu'elle soit systématiquement associée à de meilleurs résultats en lecture. La plupart des élèves ( $82 \%$ ) de l'échantillon de l'évaluation de lecture précoce étaient scolarisés avec un enseignant déclarant être de la même origine ethnique qu'eux, bien que ce pourcentage ne soit que d'environ $57 \%$ dans l'échantillon national de l'évaluation des résultats d'apprentissage des élèves. Cependant, l'absence d'impact significatif d'une scolarité avec un enseignant parlant la même langue maternelle que les élèves est quelque peu surprenant. Si l'on veut améliorer les performances scolaires des élèves, issus des minorités ethniques, il faut envisager l'utilisation des langues maternelles durant les premières années de scolarité avec un passage progressif au lao.

Nous avons également montré que les enfants issus d'un groupe ethnique minoritaire (même s'il est démographiquement important) et pauvre, ainsi que les filles vivant dans des zones rurales reculées, n'ont pas la possibilité de poursuivre leurs études au niveau secondaire inférieur ou supérieur, en raison d'un manque de capital financier ou social. Notamment, les filles vivant loin de l'école abandonnent leur scolarité pour aider leurs parents à travailler dans les rizières ou participer à l'entretien du ménage (Chounlamany, 2014).

\section{Éducation de la petite enfance}

Selon le Plan d'action national en faveur de l'éducation pour tous, le programme d'éducation et de protection de la petite enfance du Laos cherchait à atteindre un taux de scolarisation des enfants âgés de 3 à 4 ans de $11 \%$ en 2010 et de $17 \%$ en 2015. En 2013-2014, le taux d'inscription des enfants à ce niveau était de $27,0 \%$ pour les enfants âgés de 3 à 4 ans $(27,3 \%$ pour les filles et $26,8 \%$ pour les garçons). Le pays avait donc déjà atteint l'objectif fixé dans le plan national. L'augmentation du nombre de salles de classe dédiées à l'éducation de la petite enfance (de 2557 en 2006-2007 à 6837 en 2013-2014) a sans nul doute représenté un facteur favorable, mais la majeure partie de ces développements a eu lieu en zones urbaines et dans le secteur privé. Pour les enfants de 5 ans, le taux d'inscription était de 60,8 \% en 2013-2014 (60,7\% pour les garçons et $61 \%$ pour les filles) ; l'objectif fixé pour 2015 était donc également atteint (55\% au total).

\section{Redoublements et abandons dans l'enseignement primaire}

Au Laos, les taux de redoublement dans l'enseignement primaire représentent un obstacle important pour atteindre certains objectifs de l'Éducation 
pour tous et des stratégies nationales, par exemple en termes de taux de rétention et d'achèvement de l'enseignement primaire. La plupart des redoublements surviennent dès la première année du primaire. Bien que le nombre absolu de redoublants chez les élèves de première année diminue (ils étaient 85598 en 2006-2007 contre 35479 en 2013-2014), les taux de redoublement en première année du primaire sont encore importants de nos jours.

La disparité entre les sexes en termes de taux de redoublement n'est pas significative, mais l'on constate jusqu'à présent que le taux de redoublement des garçons est toujours plus élevé que celui des filles, à tous les niveaux. Les taux de redoublement varient en revanche selon les provinces. Les taux les plus bas sont ceux de la capitale et les taux les plus élevés ceux des provinces d'Attapeu, de Saravane, de Khammouane et de Phongsaly. Selon les résultats de l'enquête basée sur les indicateurs sociaux du Laos [Lao Social Indicator Survey: LSIS], ces provinces se caractérisent par un niveau de préparation à l'école et un taux net d'admission bas.

Tout comme le taux de redoublement, le taux de décrochage scolaire dans le primaire tend également à la baisse. Le taux de décrochage demeure toutefois plus élevé en première année du primaire que dans les autres niveaux du primaire. Cela s'explique notamment par les entrées précoces ou tardives en première année, par le manque de préparation au niveau primaire et par la pauvreté. Selon les conclusions de l'enquête sur les indicateurs sociaux du Laos, le taux de préparation à l'école des enfants de moins de 5 ans n'était en 2011-2012 que d'environ $24 \%$. Le taux net d'admission était de $64 \%$. En conséquence, la plupart des élèves de première année étaient des redoublants et leur taux de décrochage était également plus élevé. En outre, du fait de l'occupation de leurs parents, ces enfants étaient parfois forcés de quitter l'école, par exemple pour travailler dans les champs. Il semble néanmoins que la réduction du taux de décrochage scolaire se maintienne ces dernières années : le taux de décrochage scolaire dans l'enseignement primaire est passé de 9,4\% en 2006-2007 à 5,5\% en 2013-2014. Mais, si le taux diminue, les abandons dans le primaire demeurent importants, puisqu'environ 10000 élèves abandonnent chaque année l'école primaire.

Parmi les causes du décrochage scolaire, la culture est fréquemment citée comme influençant les décisions de scolarisation des familles. Dans les sociétés ayant de fortes normes patriarcales, la scolarisation des filles peut également être moins valorisée, voire considérée comme menaçante pour l'ordre établi. Des croyances culturelles d'ordre général sur l'importance de l'éducation peuvent également avoir un impact sur la priorité donnée par le ménage à la scolarisation des enfants et sur le soutien apporté aux enfants en dehors de l'école. Mais le décrochage est également lié aux pratiques d'éducation des enfants et à la manière dont les parents offrent un environnement stimulant à la maison, facilitant l'apprentissage et l'accès à l'école. 


\section{UNE TRIPLE EXCLUSION}

Certains soutiennent que la «triple condition » de fille, pauvre, issue d'une minorité ethnique est particulièrement préjudiciable à la fréquentation scolaire, même si les garçons issus des minorités rurales pauvres ne s'en sortent pas beaucoup mieux. Les taux d'exclusion scolaire varient fortement parmi les enfants non lao-tai des zones rurales, selon le niveau de pauvreté et l'appartenance ethnique. Les enfants pauvres non lao-tai sont environ deux fois plus susceptibles de ne jamais aller à l'école que les non pauvres, et les filles sont légèrement plus susceptibles que les garçons de ne jamais aller à l'école, quel que soit leur âge ou leur niveau de pauvreté. Le modèle est très différent pour les enfants lao-tai : l'écart entre les enfants pauvres et non pauvres est relativement plus faible, mais les garçons sont plus susceptibles de ne jamais aller à l'école que les filles (Marshall et Naka, 2016).

Environ $11 \%$ des enfants âgés de 6 à 16 ans avaient déjà été à l'école mais ne la fréquentaient plus au moment du recensement, ils ont donc été considérés comme décrocheurs. En parallèle, près de $7 \%$ des enfants âgés de 6 à 16 ans n'ont jamais fréquenté l'école. On a également constaté une proportion légèrement plus élevée d'abandons chez les filles que chez les garçons (respectivement $11 \%$ et $10 \%$ ).

L'accès aux établissements scolaires est susceptible de déterminer la probabilité qu'un enfant fréquente l'école ou non. On observe ainsi un contraste frappant dans les proportions de ceux qui n'ont jamais été à l'école chez les enfants et jeunes âgés de 6 à 25 ans, entre les zones urbaines, les zones rurales avec accès routier et les zones rurales sans accès routier : respectivement $2 \%$, $9 \%$ et $13 \%$. Des programmes gouvernementaux visant à rendre les écoles plus accessibles aux enfants dans les zones rurales sont nécessaires pour atteindre l'objectif de l'éducation primaire et secondaire universelle (Recensement du Laos, 2015).

Selon le recensement de la population du Laos de 2015, $85 \%$ de la population âgée de 15 ans et plus était alphabétisé, avec un écart important entre les sexes : le taux d'alphabétisation des femmes était de $79 \%$, contre $90 \%$ chez les hommes. Parmi les enfants âgés de 6 ans et plus, $13 \%$ n'avaient jamais fréquenté l'école, $27 \%$ la fréquentaient actuellement et $58 \%$ étaient des décrocheurs scolaires (République démocratique populaire lao, 2015).

Les résultats de l'étude menée par Jeffrey H. Marshall et Emiko Naka (2016) mettent en évidence certaines variables pertinentes. Tout d'abord, les élèves ayant déclaré avoir fréquenté une forme d'établissement préscolaire (maternelle) ne réussissent pas significativement mieux que les élèves n'en ayant pas fréquenté (selon l'évaluation de lecture précoce) et les établissements scolaires ayant une maternelle attenante obtiennent des scores plus faibles lors de l'évaluation des résultats d'apprentissage des élèves. La fréquentation préscolaire n'est un prédicteur significatif d'une maîtrise plus élevée de la lecture orale que 
lorsque les autres variables relatives à l'origine ethnique et au statut socioéconomique sont exclues. Mais le manque de signification statistique de l'éducation préscolaire dans le modèle de base complet suggère que la fréquentation préscolaire n'a pas d'effet direct et durable sur le niveau en lecture. L'interprétation des résultats concernant l'éducation préscolaire peut également être rendue complexe, du fait des nouveaux programmes ciblant les zones pauvres. Quoi qu'il en soit, l'absence de lien significatif entre éducation préscolaire et résultats d'apprentissage n'est pas inhabituel dans la littérature scientifique. Cela souligne simplement l'importance d'offrir des opportunités d'apprentissage préscolaire de qualité, car c'est le seul moyen pour les enfants de bénéficier d'avantages qui dureront tout au long de leur scolarité primaire.

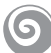

Dans cet article, nous avons tenté d'analyser le processus de construction des inégalités scolaires liées au genre et à l'appartenance ethnique. Le premier défi du Laos est d'améliorer l'accès à l'éducation des populations pauvres. Ce défi devient encore plus important pour les filles et les populations appartenant à des minorités ethniques. Ces deux groupes sont les plus susceptibles de ne pas avoir accès à l'école ou d'abandonner celle-ci d'une manière précoce. Par des mesures éducatives destinées aux populations vulnérables, le gouvernement du Laos tente d'améliorer l'équité du système éducatif. La mise en place de programmes d'éducation pour la petite enfance est l'une des mesures envisagées, même s'il faut améliorer la qualité de ces programmes avant d'escompter en recueillir les fruits sur les trajectoires scolaires des filles et des minorités ethniques.

\section{BIBLIOGRAPHIE}

CHOUNLAMANY K. (2014): An investigation of the factors to student's effective learning in rural areas of Lao PDR, Korea Foundation for Advanced Studies, Corée du Sud.

FOX C. (2003) : « No place for girls? Gender, Ethnicity and Citizenship Education in the Lao People' Democratic Republic », Compare, vol. 3., nº 3, p. 401-412.

KING E, VAN DE WALLE D. (2010) : Laos Ethno-linguistic Diversity and Disadvantage (document PowerPoint). En ligne [http://bit.ly/2LA6lSB], World Bank.

LAO PEOPLE'S DEMOCRATIC REPUBLIC (2003): Constitution of the Lao People's Democratic Republic, Vientiane: National Assembly.

LAO STATISTICS BUREAU (2016) : Results of Population and Housing Census 2015, Lao Statistics Bureau. En ligne: [https://goo.gl/XRDYTE], consulté le 19 juillet 2018.

LAO WOMEN UNION (2015) : ASEAN Regional Plan of Action on the Elimination of Violence against Women, Vientiane: Lao Women Union. En ligne [https://bit. ly/2MxbZFx], consulté le 18 août 2018. 
MARSHALL J. H., NAKA E. (2016) : Reducing Early Grade Dropout and Low Achievement in Lao PDR: Root Causes and Possible Interventions, Washington DC: Banque mondiale.

MINISTRY OF EDUCATION AND SPORTS (LAOS) (2013) : EFA-Country Report, 13th Regional EFA Coordinator Meeting, 26-27 février 2013.

(2014) : Final Evaluation Report. Teacher Training Enhancement and Status of Teachers Project (2002-2010).

(2015) : National Education for All Review Report, Vientiane.

MINISTRY OF PLANNING AND INVESTMENT (2017) : Statistical Yearbook 2016, Vientiane.

PHOUXAY K., TOLLEFSEN A. (2011) : "Rural-urban migration, economic transition, and status of female industrial workers in Lao PDR ", Population, Space and Place, $\mathrm{n}^{\circ} 17(5)$, p. 421-434. En ligne: [https://doi.org/10.1002/psp.620], consulté le 20 août 2018.

UNITED NATIONS, DEPARTMENT OF ECONOMIC AND SOCIAL AFFAIRS: UNDESA (2017) : International Migrant Stock: The 2017 Revision. En ligne [https:// bit.ly/2MvK31s] consulté le 18 août 2018.

UNESCO, UNDP, IOM, UN-HABITAT (2017) : Overview of Internal Migration in Lao PDR, Policy Briefs on Internal Migration in Southeast Asia. En ligne : [https://bit. ly/2Mn6vhz], consulté le 18 août 2018.

UNITED NATIONS IN LAO PDR (2015) : Country Analysis Report: Lao PDR. Analysis to inform the Lao People's Democratic Republic- United Nations Partnership Framework (2017-2021), Vientiane: United Nations in Lao PDR. En ligne: [https://goo.gl/ EfDhfY], consulté le 18 août 2018. 